\title{
A Method for Mill Monitoring Based on Inter-insert Periodic Correlation Using Singular Value Decomposition (SVD)
}

Xiaowen ZHU ( $\sim$ xiaowen.zhu@insa-lyon.fr)

INSA Lyon: Institut National des Sciences Appliquees de Lyon https://orcid.org/0000-0002-0868-4246

François Girardin

INSA Lyon: Institut National des Sciences Appliquees de Lyon

Jérôme Antoni

INSA Lyon: Institut National des Sciences Appliquees de Lyon

\section{Research Article}

Keywords: Condition monitoring, Milling tool, Wear, Correlation, SVD

Posted Date: November 2nd, 2021

DOI: https://doi.org/10.21203/rs.3.rs-1011007/v1

License: (c) (i) This work is licensed under a Creative Commons Attribution 4.0 International License.

Read Full License 


\title{
A Method for Mill Monitoring Based on Inter-insert Periodic Correlation Using Singular Value Decomposition (SVD)
}

\author{
Xiaowen ZHU ${ }^{1 *}$, François Girardin ${ }^{1 \dagger}$ and Jérôme Antoni ${ }^{1 \dagger}$ \\ ${ }^{1}$ Univ Lyon, INSA Lyon, LVA, EA677, 69621 Villeurbanne, \\ France.
}

*Corresponding author(s). E-mail(s): xiaowen.zhu@insa-lyon.fr; Contributing authors: francois.girardin@insa-lyon.fr; jerome.antoni@insa-lyon.fr;

†These authors contributed equally to this work.

\begin{abstract}
This paper introduces a method to monitor the wear of end milling tools in real-time production based on inter-insert periodic correlation. The aim is to detect abnormal behavior of the cutter as early as possible to prevent severe tool failure and subsequent losses. The approach takes advantage of the angular domain to segment the signal in periodic cycles of the same angular duration, which are then amenable to correlation analysis. An ordered separability index with latent correlation characteristics is proposed to assess the current operating state of the tool. A series of simulations with existing experimental data are run to test the feasibility of the proposed index and to calculate the corresponding confidence interval. This approach has a high potential to form an efficient tool condition monitoring system. Compared to the traditional teach-in method, this method is more independent of the cutting conditions (changes of velocity or direction) and has no requirement for a trial cut, making the method useful for small batch production and can reduce the rate of false alarms.
\end{abstract}

Keywords: Condition monitoring, Milling tool, Wear, Correlation, SVD 


\section{Introduction}

Computer numerical control (CNC) machining plays a vital part in today's manufacturing processes. Multiple milling techniques are widely used to pursue productivity improvement and cost-effectiveness in fields such as aerospace, aviation, and automobile manufacturing. In ideal circumstances, a machine can function for several days without stopping in a facility staffed with a small number of operators or even with an unstaffed operation. However, unplanned downtime has always been a major bottleneck that plagued industrial production.

According to statistics, cutter breakage is one of the major causes of unwanted stoppage, accounting for about $6.8 \%$ [1]. Such shutdown accidents both waste time and diminish efficiency and can cause irreparable damage to the machine (mostly to the spindle). Especially when the machined products are high added-value parts, the consequences and losses will be extremely significant if the problem is not discovered in time. The $6.8 \%$ mentioned above only refers to situations where the tool is seriously damaged. In most cases, the tool has no visible cracks but is already excessively worn. Using a dull cutter will make the machine's spindle bear greater cutting resistance, and the quality of the workpiece, such as surface roughness, will deteriorate. Therefore, monitoring the real-time status of the tool has become an essential issue in low-staff environments. An accurate and reliable tool condition monitoring (TCM) system can allow scheduled maintenance, which may save $10 \%$ to $40 \%$ of the cost of production projects [2].

Researchers have proposed many different TCM methods over the past decades. TCM can be divided into direct and indirect methods of monitoring the type and amount of wear [3]. Direct TCM usually requires a high-frequency acquisition camera $[4,5]$ or uses optical methods such as laser measurement to directly obtain visual data $[6,7]$. It is capable of capturing data on the surface, information about the cutting edge and even the geometry of the chip [8]. However, due to the practical limitation of accessibility, the lack of illumination and the presence of coolant liquid, using direct TCM is very difficult in realtime monitoring [9]. The indirect measurement method is based on various inputs, such as cutting force [10, 11], vibration [12, 13], motor current $[14,15]$, acoustic emission [16, 17], temperature [18, 19], collected by different sensors to monitor cutting in real-time. It involves the analysis of signals to determine the current work status of in-service equipment to predict possible failures.

Most indirect TCM systems rely on Teach-in \& Comparison principle methods: before formal production, several workpieces need to be machined as a trial run to obtain the standard working reference and set the thresholds of breakage detection. Hence, these TCM systems are not suitable for small-batch manufacturing and one-off productions. In addition, the detection in these systems builds upon the comparison between a reference value corresponding to the standard working condition and the real-time monitored data to assess the current working status. This approach is suited for the relatively constant (possibly oscillating but, in general, stationary) working condition. 
Unfortunately, when monitoring non-stationary operations, which are the most common tasks in the industry, these techniques provide unreliable results and often lead to numerous false alarms and unnecessary stoppages.

The research comes into this context aiming to develop a new method based on inter-insert periodic correlation using singular value decomposition (SVD) to overcome these shortages. Instead of comparing the real-time signal with a rigid reference object, the similarities and the differences inside the signal are explored. The inserts will gradually show different degrees of wear from their similar initial state over the typical tool life curve stages, and the correlation between them will continuously decrease. A literature review shows that most current research emphasizes the correlation between the proposed indicator and the mechanical state [20-22], or compares the real-time signal with a known template [23-25]. As far as the authors know, periodic segmentation of signals for correlation analysis is innovative and original.

In the case of high-speed tool rotation, the impact of the external environment change can be regarded as quasi-equivalent for all teeth. After correlation analysis, the results focus on the differences between the tool inserts. Therefore, this method can analyze end mill states under both cyclostationary (operation with constant load and straight trajectory) and cyclo-non-stationary conditions (operation with variable load and complex trajectory).

The study introduces a tool condition monitoring methodology that includes theoretical principles and an index based on the correlation analysis using SVD. Following the proposed prototype, the instrumentation setup and the data processing are specified in Section 3. Then experimental results are released to verify the index with benchmark data in different machining conditions.

Note that this work is viewed as part of a broader framework for monitoring and maintaining rotating machinery. Theoretically, this method should also have the potential to be applied to monitoring the condition of other mechanical components such as gears and bearings. In addition to using the cutting force as the data source, it can also process other signals related to the tool state, such as the instantaneous angular velocity and vibration signal.

\section{Methodology}

\subsection{Criterion principle}

End mills can only be put into machining when their edges produce parts with specified surface finish and dimensional tolerances. At the same time, tool wear is an inevitable phenomenon of the gradual loss of tool material in the contact zone between the tool and the workpiece. In actual processing, cutters experience various forms of deterioration, of which flank wear $(V b)$ is the most common [26]. Flank wear occurs at the expense of losing a portion of the sharp cutting edge, which greatly influences the quality of the product.

Differences in the amount of flank wear on individual teeth result in uneven amounts of material being removed by each tooth. From inverse deduction, 
the parameters associated with a change in chip thickness (such as cutting force and instantaneous angular speed) can be used to define a reasonable and logical cutting state criterion. For example, if the first tooth wears or breaks, the cutting condition of its corresponding process tends to be modified, and the second insert will perform a compensated overload work (Figure 1). The most obvious change is between two consecutive teeth, but changes in one tooth may also affect the third or other subsequent teeth.

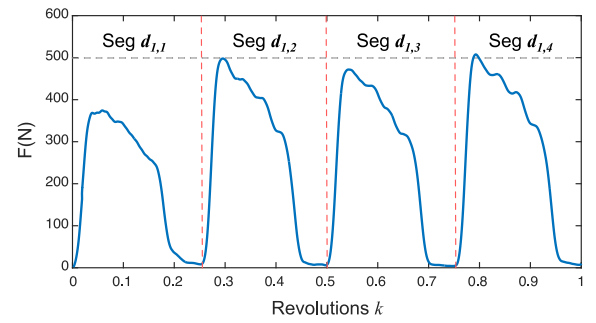

(a)

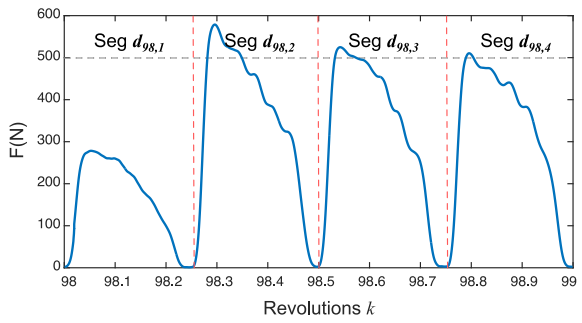

(b)

Fig. 1: Force behavior in different tool conditions: (a) initial state at $1^{\text {st }}$ revolution; (b) worn state at $98^{\text {th }}$ revolution.

The TCM system using the tool radial eccentricity proposed by Ritou et al. [27] adopted this general principle, but it only focuses on the zone of stable cuts and skips the force transients period due to path change or other factors to prevent false alarms. Among similar types of monitoring methods, most cases used the Teach-in \& Comparison principle mentioned before, which is not flexible enough to suit real-world production facilities.

To make full use of this characteristic between signal cycles, this study proposes separating the signals corresponding to different teeth and then characterizing the state of the tool by analyzing the correlation of each combination of two teeth.

\subsection{General end milling model}

\subsubsection{Force model}

This research could become a versatile method for rotating mechanisms, and the total cutting force is chosen here as an example.

According to the fundamental understanding of the end milling process, the total cutting force on a flute $F$ is composed of radial cutting force $F_{r}$ and tangential cutting force $F_{t}$. Based on the theory of Kline et al. [28], the tangential cutting force $F_{t}$ is proportional to the chip thickness $h_{c}$, and the radial force $F_{r}$ is proportional to the tangential force $F_{t}$, that is:

$$
F_{r}=K_{r} \cdot F_{t}
$$


and

$$
F_{t}=K_{t} \cdot a_{p} \cdot h_{c}
$$

where $K_{r}$ and $K_{t}$ are the cutting force coefficients contributed by the shearing action in tangential and radial directions, respectively, and are assumed to be constant for a tool-work material pair [29], and $a_{p}$ is the depth of cut.

Generally, the chip thickness is only related to the instantaneous immersion angle $\theta$ and the feed rate $f_{z}$. However, when considering tool wear phenomena, the wear amount of tooth $i$, say $V b_{i}$, and the wear amount of previous tooth $V b_{i-1}$ should also be aggregated into the chip thickness $h_{c i}$ (subscript $i$ represents the parameter corresponding to tooth $\left.i\left(i=1, \ldots, n_{z}\right)\right)$, such that

$$
h_{c}\left(\theta_{i}\right)=\left[f_{z}+\left(V b_{i-1}-V b_{i}\right)\right] \cdot \sin \theta_{i},
$$

where

$$
\theta_{i}=\theta-\frac{2 \pi}{n_{z}} \cdot i
$$

By substituting the above equations, the total cutting force corresponding to tooth $i$ can be derived as

$$
\begin{aligned}
F\left(\theta_{i}\right) & =\sqrt{\left[F_{r}\left(\theta_{i}\right)\right]^{2}+\left[F_{t}\left(\theta_{i}\right)\right]^{2}} \\
& =K_{t} \cdot a_{p} \cdot \sqrt{K_{r}^{2}+1} \cdot\left[f_{z}+\left(V b_{i-1}-V b_{i}\right)\right] \cdot \sin \theta_{i} \\
& =F_{\text {basic }}\left(\theta_{i}\right)+\Delta F\left(\theta_{i}\right) \\
& =\bar{F}+\Psi_{F}\left(\theta_{i}\right)+\Delta F\left(\theta_{i}\right),
\end{aligned}
$$

where

$$
F_{\text {basic }}\left(\theta_{i}\right)=\bar{F}+\Psi_{F}\left(\theta_{i}\right)=K_{t} \cdot a_{p} \cdot \sqrt{K_{r}^{2}+1} \cdot f_{z} \cdot \sin \theta_{i}
$$

and

$$
\Delta F\left(\theta_{i}\right)=K_{t} \cdot a_{p} \cdot \sqrt{K_{r}{ }^{2}+1} \cdot\left(V b_{i-1}-V b_{i}\right) \cdot \sin \theta_{i} .
$$

The term $F_{\text {basic }}\left(\theta_{i}\right)$ stands for the standard milling force, with $\bar{F}$ corresponding to the average force and $\Psi_{F}\left(\theta_{i}\right)$ representing the shape of the force during the cutting. $\Delta F_{i}$ embodies the part of the cutting force increased or decreased due to wear. The term $\left(V b_{i-1}-V b_{i}\right)$ actually emphasizes the similarities and differences between two adjacent teeth, which will make the subsequent process easier and also makes the results more intuitive.

\subsubsection{Geometry model}

In the process of milling, the rotation of the tool generates the removal of material on each cycle. Tool-related setting parameters (Figure 2(a)), such as cutting speed $V_{c}(\mathrm{~m} / \mathrm{min})$ (equivalent to spindle frequency $N(\mathrm{rpm})$ and $\omega$ $(\mathrm{rad} / \mathrm{s}))$, feed rate $f_{z}$, axial depth of cut $a_{p}$, and radial depth of cut $a_{e}$, will affect the operating process. At the same time, the final shape of the workpiece also largely depends on the preset milling trajectory. Because this research 
is carried out in the angular domain, changes in the trajectory will naturally introduce phase changes to the measured signal. Therefore, the spindle rotation behavior and the trajectory change behavior are parameterized into $\theta$ and $\Theta$. The geometric relationship is used to establish the model, which combines the two rotational behaviors under the same frame of reference.

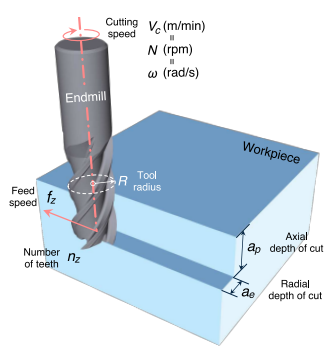

(a)

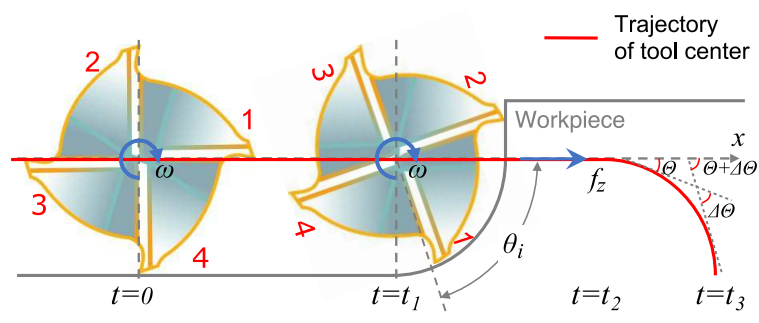

(b)

Fig. 2: End milling geometry model: (a) preset parameters; (b) preset milling trajectory.

It is assumed here that the preset trajectory is a straight line followed by a circular arc, which presents the cyclostationary and cyclo-non-stationary cases, respectively. The milling path in actual production may be more complicated, but the principle remains unchanged.

When the tool rotates, the angle $\theta$ increases along with $\omega$. Taking clockwise rotation as the positive direction, the angle of trajectory $\Theta$ is superimposed on this basis. The total angle $\vartheta$ is given by

$$
\vartheta_{i}(t)=\theta_{i}(t)+\Theta(t)=\int_{0}^{t_{3}} \omega(t) d t-\frac{2 \pi}{n_{z}} \cdot i+\Theta(t),
$$

where $\Theta$ is the angle that the tangent of the path makes with the axis $\vec{x}$ and is continuously updated by superposing the variable $\Delta \Theta$ multiple times during the milling process as indicated in Figure 2(b). The trajectory change angle $\Theta$ can be obtained from the information recorded by the axis encoder or extracted from the initial program input to the CNC machine-tool.

\subsection{Segmentation}

The purpose of segmentation is to extract the periodic cycles within the continuous sensor signal data set for further processing. In the studied case, the mechanism of the previous tooth entering and then leaving the material followed by an empty gap until the next tooth enters again is divided into periodic segments as shown in Figure 1. Note that the radial depth of cut $a_{e}$ in the experiment has been controlled to ensure that only one tooth is in contact with the material at a time to clearly illustrate the processing results. However, it 
is envisioned that this method will also be applicable even if more than one tooth is in contact with the material because the periodicity of the signal still exists, and one revolution always contains $n_{z}$ segments.

In this work, the data generated by one tool revolution is selected as the calculation unit to reduce the amount of calculation while following the tool dynamics in real time. The matrix $\mathbb{D}_{\mathbb{k}} \in \mathbb{R}^{m \times n_{z}}$ contains the data set of experiments in $k^{t h}$ revolution, which can be expressed as

$$
\mathbb{D}_{\mathbb{k}}=\left[\begin{array}{llllll}
\mid & \mid & & & \mid \\
d_{k 1} & d_{k 2} & \cdots & d_{k i} & \cdots & d_{k n_{z}} \\
\mid & \mid & & \mid & & \mid
\end{array}\right],
$$

where $\boldsymbol{d}_{\boldsymbol{k} i}$ is the $i^{t h}\left(i=1,2 \ldots n_{z}\right)$ segment sequence in the $k^{t h}$ revolution. Each sequence is composed of $m$ data points corresponding to the milling signature of each tooth. This matrix is updated by each revolution continuously, and it always contains the relevant data to differentiate and compare multiple teeth passes of each cycle.

According to the theory in Section 2.2.1, $\boldsymbol{d}_{\boldsymbol{k} i}$ corresponds to the cutting force $F\left(\theta_{i}\right)$ (equation 5); the signal segments can be further expressed as

$$
\boldsymbol{d}_{\boldsymbol{k} i}=\overline{d_{k i}} \cdot \mathbf{1}_{m, 1}+\boldsymbol{\Psi}_{\boldsymbol{F}_{\boldsymbol{k} i}}+\boldsymbol{\Delta} \boldsymbol{F}_{\boldsymbol{k} \boldsymbol{i}}
$$

where $\overline{d_{k i}}$ is the average value corresponding to the $i^{\text {th }}$ segment in $k^{\text {th }}$ revolution $\boldsymbol{d}_{\boldsymbol{k} \boldsymbol{i}}$, and $\mathbf{1}_{m, 1}$ is an all-ones vector with dimension $m \times 1$. The term $\boldsymbol{\Psi}_{\boldsymbol{F}_{\boldsymbol{k} \boldsymbol{i}}}$ corresponds to the mean (theoretical) shape of the zero-centered cutting force. The term $\boldsymbol{\Delta} \boldsymbol{F}_{\boldsymbol{k} \boldsymbol{i}}$ presents the chip thickness characteristic due to the individual tooth condition.

When the $n_{z}$ teeth have the same state, the sequences possess a remarkably close signal signature so that the ideal revolution matrix $\mathbb{D}_{\mathbb{k}}$ can be written as

$$
\text { Ideal } \mathbb{D}_{\mathbb{k}}=\overline{d_{k i}} \cdot \mathbb{1}_{m, n_{z}}+\left(\boldsymbol{\Psi}_{\boldsymbol{F}_{\boldsymbol{k} \boldsymbol{i}}}+\boldsymbol{\Delta} \boldsymbol{F}_{\boldsymbol{k} \boldsymbol{i}}\right) \cdot \mathbb{1}_{1, n_{z}},
$$

However, with the real-world external disturbances that are difficult to exclude, the revolution matrix $\mathbb{D}_{\mathbb{k}}$ will perform differently as

$$
\begin{gathered}
\mathbb{D}_{\mathbb{k}}=\overline{D_{k}} \cdot \mathbb{1}_{m, n_{z}}+\left(\boldsymbol{\Psi}_{\boldsymbol{F}_{\boldsymbol{k}}}+\boldsymbol{\Delta} \boldsymbol{F}_{\boldsymbol{k}}\right) \cdot \mathbb{1}_{1, n_{z}}, \\
\mathbb{D}_{\mathbb{k}}=\overline{D_{k}} \cdot \mathbb{J}+\mathbb{\Downarrow}_{F_{k}}+\Delta F_{k},
\end{gathered}
$$

where $\mathbb{J}=\mathbb{1}_{m, n_{z}}$. The average value $\overline{D_{k}}$, the theoretical shape $\boldsymbol{\Psi}_{\boldsymbol{F}_{\boldsymbol{k}}}$ and the remaining individual residual $\boldsymbol{\Delta} \boldsymbol{F}_{\boldsymbol{k}}$ extracted by $\mathbb{D}_{\mathbb{k}}$ are not exactly the same as $\overline{d_{k i}}, \boldsymbol{\Psi}_{\boldsymbol{F}_{\boldsymbol{k} \boldsymbol{i}}}$ and $\boldsymbol{\Delta} \boldsymbol{F}_{\boldsymbol{k} \boldsymbol{i}}$. However, they should be very similar (within a certain error). In addition, the difference between them increases significantly when the teeth gradually show wear. Considering that the chip thickness characteristic due to individual tooth condition $\Delta F_{k}$ is relatively much smaller than the original value of the data, if removing $\overline{D_{k}} \downarrow$ to reach zero-centering, then the contribution of $\Delta F_{k i}$ will be more prominent, and the subsequent analysis results will be more obvious. The segmentation is programmed automatically by the milling parameters preset by the user in the CNC machine tool. 


\subsection{Correlation}

In probability and statistics, correlation is essentially the measure of how two or more sequences are related to one another with the value of coefficient between -1 and 1 . The signal segments divided in Section 2.3 have causal links as described in Section 2.1. Therefore, the correlation can indicate a predictive relationship that can be exploited in a TCM system.

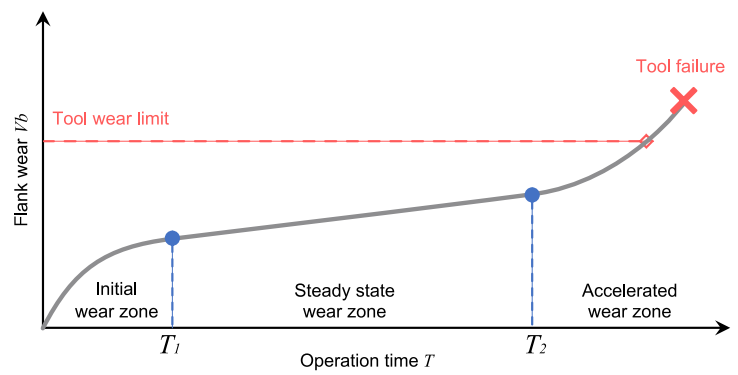

Fig. 3: Tool life $(T)$ curves: variation of flank wear $(V b)$ land with operation time.

The initial length difference between the teeth of the new end mill is very small, within a few hundred millimeters. According to the typical tool life curve in Figure 3, a very sharp edge of the tool is worn soon after the cutting starts [29]. Each insert of the end mill will experience a varying extent of wear in the initial stage. At this time, the correlation among the teeth will decrease slightly. This is followed by an approximately linear tool wear period, in which the correlation coefficient remains at a stable value. After the wear land reaches a critical limit, flank wear increases exponentially, which leads to a cliff-drop of the correlation, and a warning will be given when the correlation coefficient falls below the threshold. Because the correlation analysis is performed on the real-time parameters, the proposed index will fluctuate but will not exceed the threshold when passing through a changing path such as a curve, compared with the large change of the original signal. Therefore, false alarms can be avoided.

Traditional correlation analysis, such as Pearson's correlation coefficient or the Kendall rank correlation coefficient, is a statistical relationship between two random variables [30]. However, the tooth number $n_{z}$ of the common endmill is often 2 to 6 or more. Therefore, if the traditional method is used to calculate the real-time correlation coefficient of any combination of two teeth, a large number of local comparisons will be produced, but no intuitive judgement of the bigger picture can be drawn.

To assess the dependence between several variables at the same time, the correlation matrices $\left(\mathbb{D}_{\mathbb{k}}-\overline{D_{k}} \mathbb{l}\right)\left(\mathbb{D}_{\mathbb{k}}-\overline{D_{k}} \mathbb{l}\right)^{T}$ and $\left(\mathbb{D}_{\mathbb{k}}-\overline{D_{k}} \mathbb{l}\right)^{T}\left(\mathbb{D}_{\mathbb{k}}-\overline{D_{k}} \mathbb{l}\right)$ 
are considered, whose results contain the correlation coefficients between each variable and the others.

\subsection{Singular Value Decomposition (SVD)}

Singular value decomposition (SVD) is selected as the tool to continue the subsequent processing to benefit from the advantage of component identification and, at the same time, satisfy the correlation matrix analysis requirement. The following results show that SVD can meet the requirements.

SVD is commonly used in applications such as big data analysis or image compression [31]. It extracts the main components from a large amount of data using underlying principle of correlation analysis. It provides a systematic interpretation in terms of correlation among the columns and the rows of $\left(\mathbb{D}_{\mathbb{k}}-\overline{D_{k}} \mathbb{J}\right)$. Whether $n_{z}$ is greater than $m$ or $m$ is greater than $n_{z}$ (assuming $n_{z}<m$ in the following discussion), the SVD always has a unique matrix decomposition that exists for every input matrix $\left(\mathbb{D}_{\mathbb{k}}-\overline{D_{k}} \mathbb{J}\right)$. The data set can be decomposed into three parts as

$$
\begin{aligned}
& \mathbb{D}_{\mathbb{k}}-\overline{D_{k}} \mathbb{J}=\mathbb{U}_{\mathbb{k}} \mathbb{Z}_{\mathbb{k}} \mathbb{V}_{\mathbb{k}}^{\mathbb{T}} \\
& =\left[\begin{array}{llll}
\mid & \mid & & \mid \\
\boldsymbol{u}_{k 1} & \boldsymbol{u}_{k 2} & \cdots & \boldsymbol{u}_{k m} \\
\mid & \mid & & \mid
\end{array}\right] \cdot\left[\begin{array}{ccc}
\sigma_{k 1} & & \\
& \ddots & \\
& & \sigma_{k n_{z}} \\
- & - & - \\
& 0 &
\end{array}\right] \cdot\left[\begin{array}{ccc}
-\boldsymbol{v}_{\boldsymbol{k} \mathbf{1}} \boldsymbol{T}^{\boldsymbol{T}} & - \\
-\boldsymbol{v}_{\boldsymbol{k} \mathbf{2}}{ }^{2} & - \\
\vdots & \\
-\boldsymbol{v}_{\boldsymbol{k n} \boldsymbol{n}_{\boldsymbol{z}}} \boldsymbol{T} & -
\end{array}\right]
\end{aligned}
$$

where $\mathbb{U}_{\mathbb{k}} \in \mathbb{R}^{m \times m}$ and $\mathbb{V}_{\mathbb{k}} \in \mathbb{R}^{n_{z} \times n_{z}}$ are unitary matrices, and $\mathbb{Z}_{\mathbb{k}} \in \mathbb{R}^{m \times n_{z}}$ is a matrix with real, non-negative entries on the diagonal and zeros off the diagonal. Here ${ }^{T}$ denotes the complex conjugate transpose.

If taking this decomposition into a physical explanation without looking at the specific application scenarios, then $\mathbb{U}_{\mathbb{k}}$ is the hierarchically arranged "eigen" information about the measurements, and $\mathbb{V}_{\mathbb{k}}$ is essentially the "eigen" time series that stands for how each mode $\mathbb{V}_{\mathbb{k}}$ evolves in the duration of the process. $\mathbb{Z}_{\mathbb{k}}$ represents the amount of energy that each of these column vectors captures, hierarchically arranged in order of importance.

For a better understanding, one can simplify Equation (14) in the following way:

$$
\mathbb{D}_{\mathbb{k}}-\overline{D_{k}} \rrbracket=\sigma_{k 1} \boldsymbol{u}_{\boldsymbol{k} \mathbf{1}} \boldsymbol{v}_{\boldsymbol{k} \mathbf{1}}^{\boldsymbol{T}}+\sigma_{k 2} \boldsymbol{u}_{\boldsymbol{k} 2} \boldsymbol{v}_{\boldsymbol{k} \mathbf{2}}^{\boldsymbol{T}}+\ldots+\sigma_{k n_{z}} \boldsymbol{u}_{\boldsymbol{k n} n_{z}} \boldsymbol{v}_{\boldsymbol{k} n_{z}}^{\boldsymbol{T}}
$$

Here, $\sigma_{k i}$ are the ordered singular values, $\boldsymbol{u}_{\boldsymbol{k} \boldsymbol{i}}$ is an $m$-dimensional column vector, and $\boldsymbol{v}_{\boldsymbol{k} \boldsymbol{i}}$ is an $n$-dimensional column vector. The matrix $\left(\mathbb{D}_{\mathbb{k}}-\overline{D_{k}} \downarrow\right)$ is decomposed by SVD into a weighted, ordered sum of separable matrices $\sigma_{k i} \boldsymbol{u}_{\boldsymbol{k} \boldsymbol{i}} \boldsymbol{v}_{\boldsymbol{k} \boldsymbol{i}}^{\boldsymbol{T}}$. To estimate the ability of the $i^{\text {th }}$ order singular value to 
approximate the original matrix, the following separability index

$$
\alpha_{k i}=\frac{\sigma_{k i}^{2}}{\sum_{j=1}^{n} \sigma_{k j}^{2}},
$$

is put forward to precisely indicate the nature of its separability [31]. The proposed separability index can measure the proportion of the $i^{\text {th }}$ order component to the set of all components in the decomposition process. Note that in the $\mathbb{D}_{\mathbb{k}}$ matrix, the subscript $i$ represents the number of the corresponding segment, whereas after SVD, the subscript $i$ represents the order of the hierarchical decomposition. The specific physical meaning corresponding to the three obtained parts $\mathbb{U}_{\mathbb{k}}, \mathbb{Z}_{\mathbb{k}}, \mathbb{V}_{\mathbb{k}}^{\mathbb{T}}$ will be explained in detail in Section 4.1.

\section{Experimental validation}

\subsection{Material}

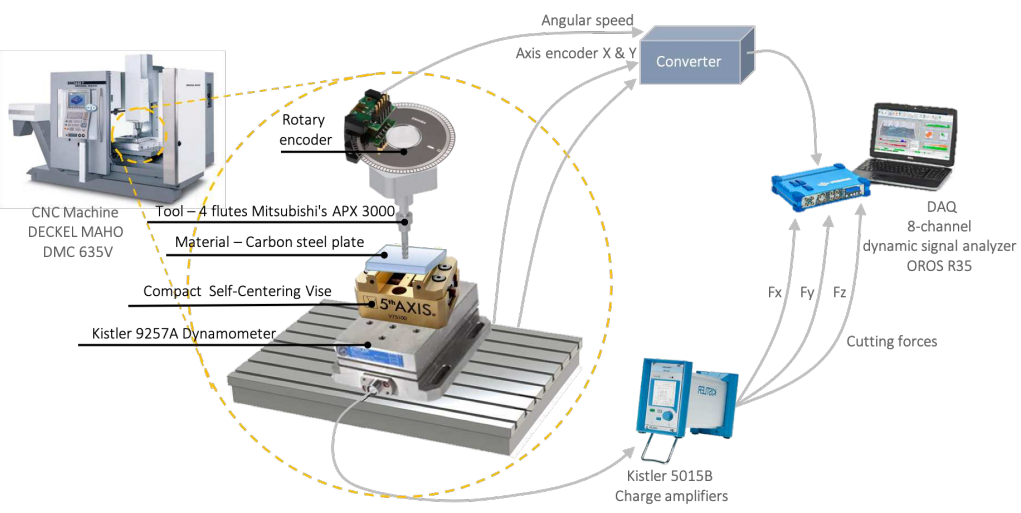

Fig. 4: Experimental setup

The DECKEL MAHO DMC 635 V milling machine was used for the experimental tests. A Kistler 9257A dynamometer table was mounted under the workpiece; after coupling with Kistler 5015B charge amplifiers, the cutting forces are measured in three orthogonal directions $(\vec{x}, \vec{y}, \vec{z})$. Simultaneously, two axis encoders for $\vec{x}, \vec{y}$ directions integrated into the bench and the rotational encoder combined in the spindle were linked to an output signals converter. The cutting force signal and encoder information are acquired synchronously, and all data converge into the Oros R35 8-channel dynamic signal analyzer. The signal acquisition process is illustrated in Figure 4.

The tool used in the experiment comes from Mitsubishi's APX 3000 series with four flutes. After the mounting of the VP15TF coated inserts, the operating diameter reaches $32 \mathrm{~mm}$. The workpiece used for milling was a C45 medium 
carbon steel plate with a dimension of $20 \times 100 \times 100 \mathrm{~mm}$. The cutting speed of $140 \mathrm{~m} / \mathrm{min}$ and the sampling frequency of $50 \mathrm{kHz}$ allows collecting 8-9 sample points for each sine cycle of spindle encoder signal. The specific cutting parameters and cutting path are as displayed in Table 1 and Figure 5.

\begin{tabular}{lll}
\hline Notation & Parameter & Value \\
\hline$n_{z}$ & Number of teeth & 4 \\
$N(\mathrm{rpm})$ & Spindle frequency & 1393 \\
$V_{c}(\mathrm{~m} / \mathrm{min})$ & Cutting speed & 140 \\
$f_{z}(\mathrm{~m} / \mathrm{rev})$ & Feed speed & 0.1 \\
$a_{e}(\mathrm{~mm})$ & Radial depth of cut & $3.2,8,12$ \\
$a_{p}(\mathrm{~mm})$ & Axial depth of cut & 3 \\
\hline
\end{tabular}

Table 1: Cutting conditions

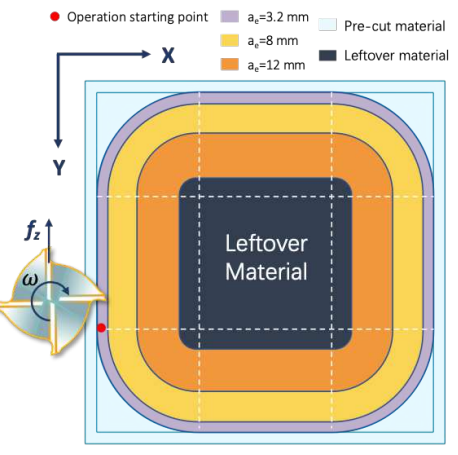

Fig. 5: Cutting path

The four corners of the workpiece have been removed in advance during the pretreatment. The purple, yellow, and orange areas correspond to three passages in different engagements of the cut, $a_{e}=3.2,8,12 \mathrm{~mm}$, respectively. The milling type is down-milling, and the operation always starts at the same point.

\subsection{Signal processing}

The study proposes a methodology for assessing the tool condition by exploiting the correlation between teeth signatures. Useful information, hidden fairly deep in the raw signal, includes the preceding and following idle rotations of the end-mill at the settled spindle speed. The key steps of the signal processing are described below:

\section{(i) Filtering}

The built-in rotary encoder of the spindle emits 256 pulses per revolution. The main frequency is the spindle frequency multiplied by the encoder resolution. A low-pass Butterworth filter is applied with the cut-off frequency set to 1.5 times the main frequency to improve the signal-tonoise ratio. In this way, the high-frequency noise is removed.

(ii) Resampling in the angular domain

The dynamometer under the workpiece records the force change over time, while the rotary encoder registers the information of the corresponding angle domain. Resampling the signal from the time domain to the angular domain is a very important step. 


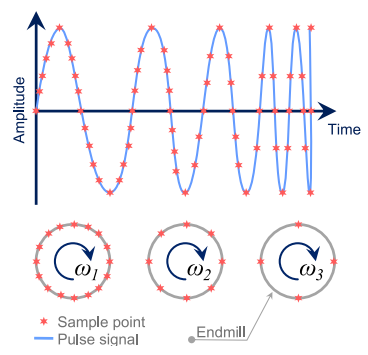

(a)

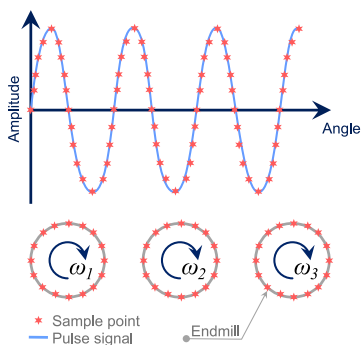

(b)

Fig. 6: Schematic diagram for data acquisition under different domains with variable rotational speed $\left(\omega_{1}<\omega_{2}<\omega_{3}\right)$ : (a) the number of samples collected per revolution varies with the rotational speed if sampling in the time domain; (b) the number of samples collected per revolution is evenly distributed regardless of rotational speed if sampling in the angular domain.

From a macro point of view, the cutting speed has been set at a fixed value, but as a matter of fact, due to cutting force/torque change and servo control, the milling speed changes constantly and slightly. This leads to differences in the number of sampling points within the same time length, making it difficult to later divide the signal into segments corresponding to each tooth. Compared with time sampling, the resampling in the angular domain can stabilize the number of samples per revolution, regardless of the rotation speed. As shown in Figure 6, no matter how the cutting speed changes $\left(\omega_{1}<\omega_{2}<\omega_{3}\right)$, a constant number of sampling points will be recorded for each revolution.

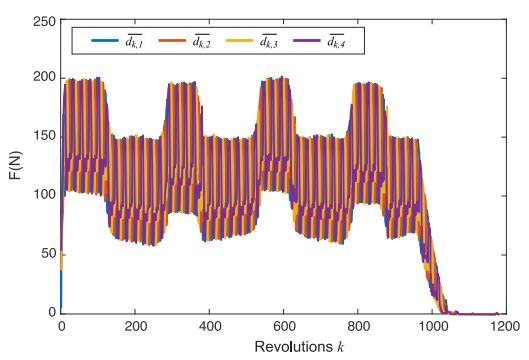

(a)

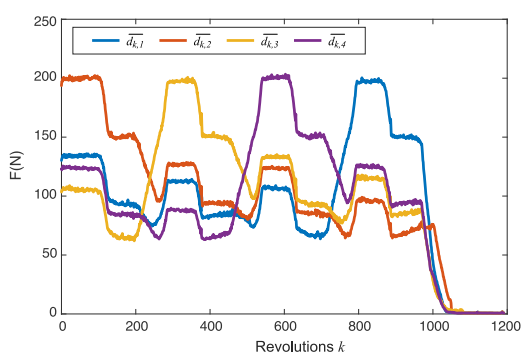

(b)

Fig. 7: Comparison of the average value of each segment $\overline{d_{k i}}$ before and after resampling in the angular domain: (a) sampling in the time domain; (b) resampling in the angular domain $(\theta)$

The average value of segment $\overline{d_{k i}}$, as defined in Section 2.3, can be used to roughly represent the general state of the segment. By connecting $\overline{d_{k i}}$ 
with the same segment $i$ in series, the results provide a principal tendency to observe briefly whether the subsequent optimization process is effective before the introduction of correlation analysis.

As shown in Figure 7, the data with the same subscript $i$ are plotted as one line with the rotation cycles as the abscissa. The results before and after resampling are strongly affected by whether the signal is considered in the time or in the angular domain.

(iii) Milling direction correction

This work ensures that the force signal is correctly synchronized with the tooth signatures. When cutting in a straight line, only angular interpolation is needed to achieve this goal. However, changing the cutting path will naturally introduce a phase shift, which affects the distribution of the signal to the cutting edge. Fortunately, the trajectory change can be simply corrected through the combination of the tool rotation angle $\theta$ and the trajectory change angle $\Theta$ as mentioned in Section 2.2.2.

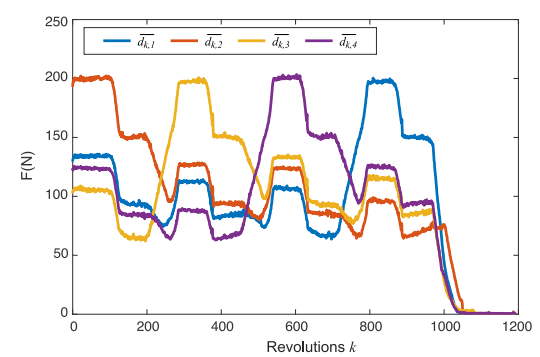

(a)

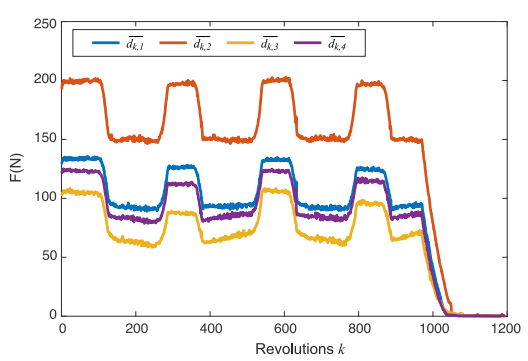

(b)

Fig. 8: Comparison before and after trajectory correction by the average value of each segment $\overline{d_{k i}}$ : (a) angle of reference $(\theta)$ without correction; (b) angle of reference $(\theta+\Theta)$ with correction.

After the correction, the signal is shifted to the position corresponding to the correct segment. The cutting force is stable at a high value during the straight cutting section and drops to a low point when entering the arc. The cycle repeats four times along the trajectory (Figure 8). In traditional monitoring, similar parameter changes are likely to cause false alarms, whereas, in the proposed method, the impact resulting from trajectory changes can be minimized after correlation analysis because the segments have undergone identical milling changes almost simultaneously. Due to the limitations of experimental circumstances, the tools used are not brand new. The second tooth is about $0.06 \mathrm{~mm}$ longer than the other teeth at the beginning of the experiment. Therefore every time it cuts into the material, the force it receives is about $50 \mathrm{~N}$ stronger than the other teeth. 
This difference is within the allowable range of machining accuracy.

\section{(iv) Truncation $\&$ segmentation $\&$ zero-centering}

The idling component of the signal (before the tool enters into the material) is of no value for identifying the signal signature corresponding to each segment. Hence, this part is truncated by manual selection. The remaining signal is segmented as described in Section 2.3. The original one-column data sequence is folded into $k$ matrices, each of which has $n_{z}$ columns containing $m$ points.

As mentioned in Section 2.2.1 and derived in Equation 13, the milling model is composed of three parts: the average value and the mean shape of the standard cutting force are determined by the original tool state and the superimposed force $\Delta F_{k}$ (increased or decreased) due to the condition of the edge. The resulting change is very small compared to the original value; it needs to be centralized before correlation analysis. Zero-centering refers to the matrix $\left(\mathbb{D}_{\mathbb{k}}-\overline{D_{k}} \mathbb{J}\right)$. In this way, the performance of relevance will be emphasized, and this method can be applied to higher-precision monitoring. The experimental results show that zero-centering can also remove the random effects in the shutdown process.

(v) SVD processing

After zero-centering, SVD will provide a hierarchical representation of the data by dominant correlations within the data. The SVD decomposition process and the separability index are recalculated at each revolution to master the real-time condition, as illustrated in Figure 9.

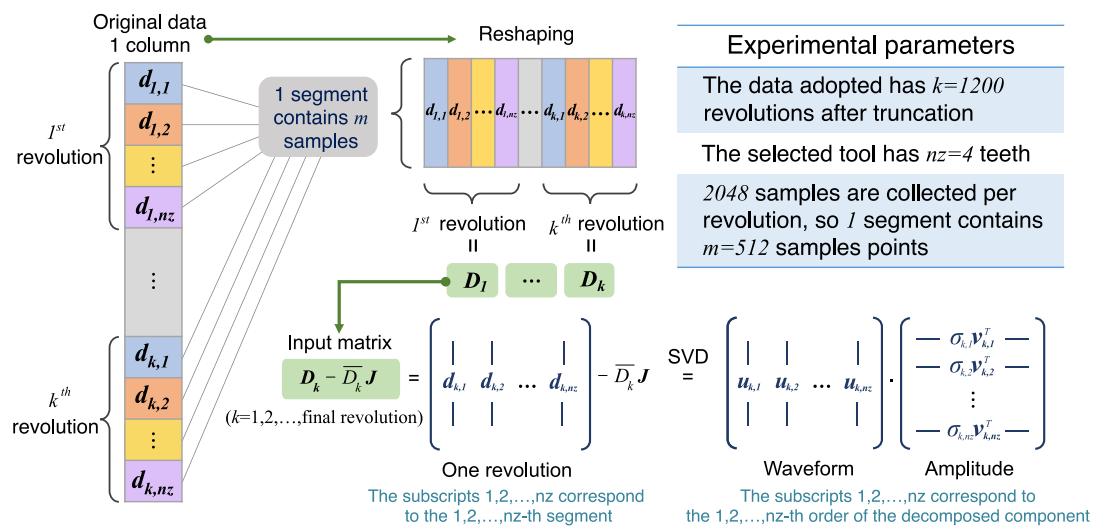

Fig. 9: Data processing flow

In the current case, the four-tooth end mill rotates for a total of 1200 revolutions and exits the workpiece at around $1050^{\text {th }}$ revolutions. Thanks 
to the resampling in the angular domain, each revolution contains exactly 2048 samples, which is equivalent to 512 points per segment.

\section{Analysis of results}

The results of three contour milling experiments with $a_{e}=3.2,8,12 \mathrm{~mm}$ are almost the same, and the state of the tool (flank wear) before and after milling remains basically unchanged. Therefore, the experimental data set with $a_{e}=$ $3.2 \mathrm{~mm}$ is used as a basis for showing the status of health tools.

To verify the performance of the proposed methodology under the worn tool situation, a milling data set is borrowed from F. Girardin et al. [32] for experimental validation of failure detection.

\subsection{SVD components}

After SVD is performed on the data of each revolution, the decomposition results obtained are presented in the following three-dimensional graphs.

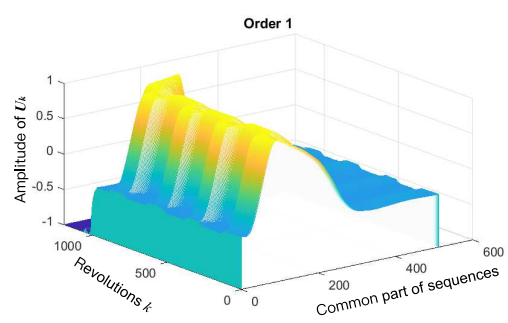

(a)

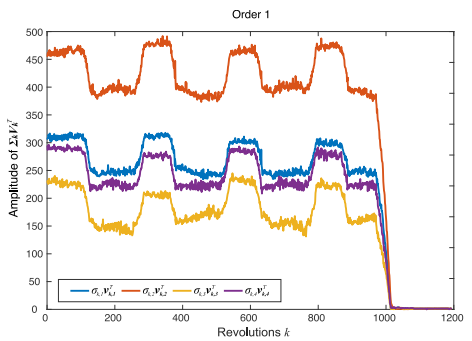

(b)

Fig. 10: SVD results for a tool in good condition: (a) left singular vector $\boldsymbol{u}_{\boldsymbol{k}}$ of SVD as a function of revolutions presents a basic signal waveform; (b) ordered singular value $\sigma_{k}$ times transposed right singular vector $\boldsymbol{v}_{\boldsymbol{k}}^{\boldsymbol{T}}$ presents the corresponding force value

The left singular vector $\boldsymbol{u}_{\boldsymbol{k}}$ extracts the common part contributed mainly by $\Downarrow_{F_{k}}$ (defined in Equation $\left.(13)\right)$ for each input matrix $\left(\mathbb{D}_{\mathbb{k}}-\overline{D_{k}} \mathbb{l}\right)$. It can be seen from Figure 10(a) that the first-order result of a single revolution is displayed with 512 sample points that form a basic waveform of $k^{t h}$ revolution. Throughout the milling process, the waveform remains unchanged, but there is a slight phase shift towards the right when the cutter passes through the arc. This is the phase shift that is naturally introduced by the cutting path mentioned in Section 3.2 (iii). The milling direction correction in signal processing has greatly improved the situation of phase change, but a residue remains.

The right singular vector $\boldsymbol{v}_{\boldsymbol{k}}$ contains the amplitude ratio corresponding to the $n_{z}$ segments within $\left(\mathbb{D}_{\mathbb{k}}-\overline{D_{k}} \mathbb{l}\right)$, which reflects the influence of $\boldsymbol{\Delta} \boldsymbol{F}_{\boldsymbol{k}}$. The 
ordered singular value $\sigma_{k}$ is the amplification factor. The combination of the above two terms $\sigma_{k} \boldsymbol{v}_{\boldsymbol{k}}{ }^{\boldsymbol{T}}$ represents the characteristic amplitude value for the corresponding force of each insert after zero-centering.

One needs to pay attention to the difference between Figure 10(b) and Figure 8(b). Figure 8(b) simply reports the speed sequence of each segment into a single average value $\overline{d_{k i}}$, while Figure $10(\mathrm{~b})$ presents the characteristic amplitude extracted from the matrix $\left(\mathbb{D}_{k}-\overline{D_{k}} \mathfrak{J}\right)$, which can preserve the signal information as much as possible by combination with the left singular vector $u_{k}$.
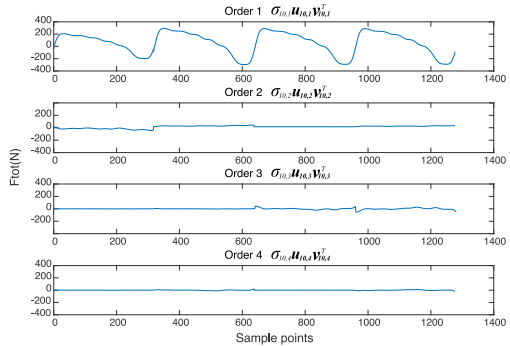

(a)

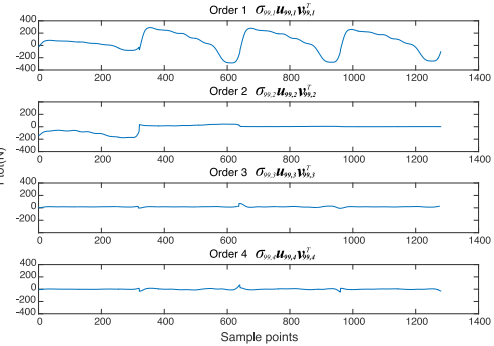

(b)

Fig. 11: Hierarchical presentation of signal by order: (a) the $1^{\text {st }}$-order component occupied the dominant contribution at the beginning of tool wear; (b) when a tooth is severely worn, the $1^{s t}$-order components are not enough to express the signal signature; thereby, the proportion of the $2^{\text {nd }}$-order components increases.

SVD provides hierarchical results from the $1^{\text {st }}$ order to the $n_{z}{ }^{\text {th }}$ order (Equation (15)), which reserves more information for detailed analysis. Using the data set from Girardin et al. as an example, the $1^{\text {st }}$ order shown in Figure 11 is the closest in the mean-square sense to the signals, and the $2^{\text {nd }}$ order or higher order can supplement the remaining details on this basis. Figure 11(a) presents the tool state of the $10^{\text {th }}$ revolution where tool wear begins, whereas Figure 11(b) indicates the behavior in the $99^{\text {th }}$ revolution where the first tooth is completely worn.

\subsection{Separability index}

The $1^{\text {st }}$ order of the separability index $\alpha_{k 1}$ remains above $90 \%$ or more (Figure 12 ), whereas the $2^{\text {nd }}, 3^{\text {rd }}$, and $4^{\text {th }}$ orders are not dominant. This means that the cutting conditions of the four teeth have a fairly high similarity throughout the whole operation process, and the decomposed $1^{s t}$-order component represents most of the information. This state is quite stable. During the trajectory change, the index slightly fluctuates, corresponding to milling condition adjustments, except that there is a small falling gap during the shutdown process. 


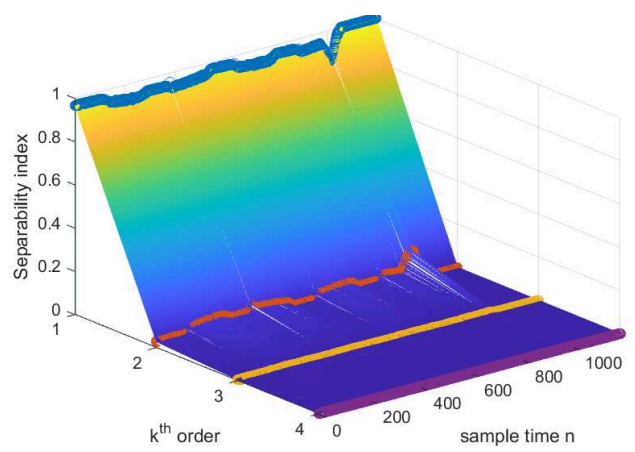

Fig. 12: Separability index for each order with respect to samples

This is a reasonable adjustment caused by the withdraw of the tool. When the workpiece completely leaves the material, the subsequent idling part has no cutting resistance, so the separability index $\alpha_{k 1}$ is closer to 1 than during milling operation.

\subsection{Sensitivity analysis}

In the above-mentioned signal processing (Section 3.2 (iv)), the preceding idling part is truncated to accurately select the starting point of the segmentation. This step ensures that each tooth signature corresponds to the segment, rather than one continuous signature being divided into two segments. However, it is a relatively ideal situation achieved by manual choice. The data length of the idling part is variable when the proposed method is implemented in real-time monitoring with different milling items. Therefore, it is not realistic to expect an accurate and automatic division of the signal at the starting position of the cutting force every time. The concept of window shift sensitivity range $\Delta_{W S}$ is introduced to proceed with sensitivity analysis and include this uncertainty in the input.

As named, it is assumed to be a shifted window of size $m$ sliding backward from the beginning of the original one-column data sequence to capture the sample set corresponding to the first segment $\boldsymbol{d}_{\mathbf{1 1}}$, which can be expressed as

$$
\boldsymbol{d}_{\mathbf{1 1}}=\left[\begin{array}{c}
d_{1+(m-l)(L-1)} \\
d_{2+(m-l)(L-1)} \\
\vdots \\
d_{m+(m-l)(L-1)}
\end{array}\right],
$$

where

$$
L=\left\lceil\frac{m}{m-l}\right\rceil \text {. }
$$

The scalar $d$ represents a sample point, and the subscript shows the row number of the sample in the original one-column data set. $l(l \in \mathbb{N})$ is the overlap 
number in the shifting process. $L$ is the execution number of the shift defined by the ceiling function of $\frac{m}{m-l}$. Once the first segment is chosen, the following data points will fold into input matrices and proceed with correlation analysis as normal steps for each shift. The process of element shifting for the general segment is described as

$$
\boldsymbol{d}_{\boldsymbol{k} \boldsymbol{i}}=\left[\begin{array}{c}
d_{m(k i-1)+1+(m-l)(L-1)} \\
d_{m(k i-1)+2+(m-l)(L-1)} \\
\vdots \\
d_{m k i+(m-l)(L-1)}
\end{array}\right] .
$$

By presetting the overlap points $l$, the means of the window shift can achieve an approximate ergodicity $(l>95 \% \mathrm{~m})$. The separability indexes of multiple processes corresponding to different start points will form the value range $\Delta_{W S}$. This indicator reflects how the separability index is affected by the choice of the starting point of the data.

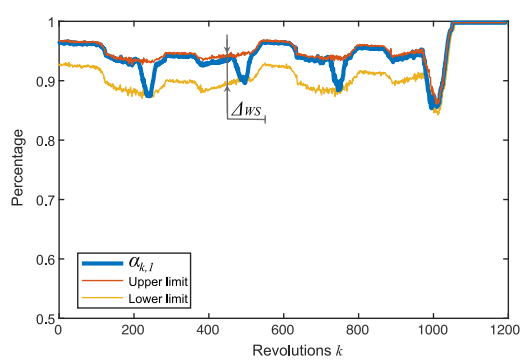

(a)

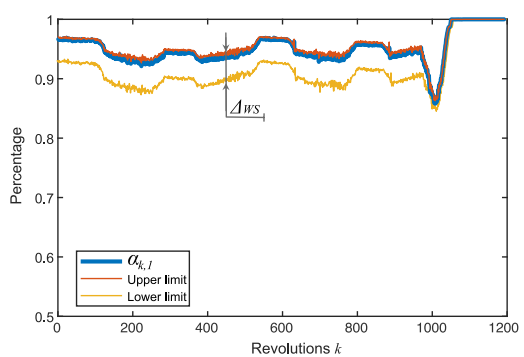

(b)

Fig. 13: Sensitivity analysis $\Delta_{W S}$ of separability index $\alpha_{k 1}$ before and after trajectory correction is almost the same: (a) angle of reference $(\theta)$ without correction; (b) angle of reference $(\theta+\Theta)$ with correction.

In the previous text (Equation (8)), the behavior of the signal is defined as a function of the rotational angle corrected with trajectory angle $(\theta+\Theta)$. The separability index $\alpha_{1}$ is greatly influenced by the combined angle. It can be seen that the data without trajectory correction have jitters during each passage of corner (Figure 13(a)), whereas the lines become smoother after correction (Figure 13(b)). When performing the sensitivity analysis, each shift will introduce an additional angle $\theta_{c}$, which varies according to the sliding window position. After $\theta_{c}$ goes from 0 to $\frac{2 \pi}{n_{z}},\left(\theta+\left(\Theta+\theta_{c}\right)\right)$ can be seen as a set that contains all the possible gyration angles including the trajectory angle $\Theta$. Thereby the separability index must be included in the sensitivity range $\Delta_{W S}$ regardless of the trajectory correction (Figure 13). Note that the upper and the lower limits of the sensitivity range $\Delta_{W S}$ possess a very similar tendency, and the value of $\Delta_{W S}$ is nearly constant. That means that the possible results 
obtained from all gyration angles are consistent for each revolution, indicating that no tool condition problem interfered during the operation. After comparing multiple sets of data, $\Delta_{W S}$ will fluctuate to a certain extent according to different cutting parameters, but the value of $\Delta_{W S}$ can be considered constant under the same cutting conditions (the error between Figure 13(a) and Figure $13(\mathrm{~b})$ is about $0.0047 \pm 0.001$ ). If the starting point is well selected, the separability index basically coincides with the upper limit of $\Delta_{W S}$.

\subsection{Fault detection}

Based on the above results, two methods are derived for assessing the status of the tool:

(i) TCM based on separability index $\alpha_{k 1}$

The judgment directly made by the performance of the separability coefficient is clear and intuitive. Under normal operating conditions, the $1^{\text {st }}$-order main components can already explain more than $90 \%$ of the information. In the event of abnormal wear, the signal will deviate from the typical waveform, and the $1^{\text {st }}$ order will no longer be sufficient to describe the signal, while the $2^{\text {nd }}$ - or $3^{r d}$-order contribution of SVD will increase rapidly.

The milling data borrowed from F. Girardin et al. [32] consist of tool state information. The tool is initially in an uncertain state - it has already been used - and breaks during testing. The tool gradually wears out, and a tooth breaks during the $94^{\text {th }}$ revolution of the spindle. If the results obtained are consistent with the known tool state after processing this set of data according to the steps in Section 3.2, the effectiveness of the proposed method can be verified.

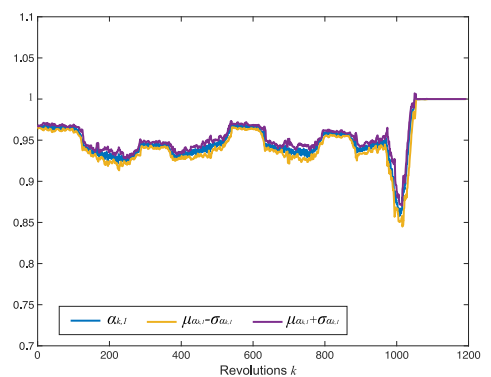

(a)

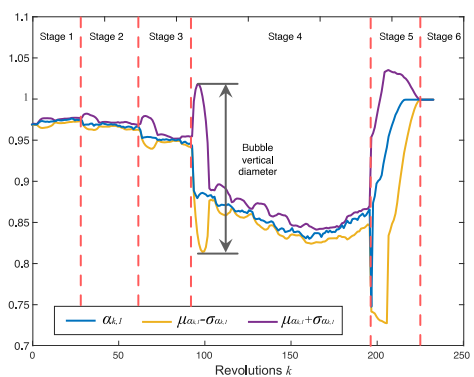

(b)

Fig. 14: Fault detection based on separability index $\alpha_{k 1}$ (blue line) with upper (purple line) and lower (yellow line) limit of 3-sigma interval: (a) tool in good condition; (b) worn tool. 
For tools in good condition, the separability index is always above 0.9 before the stoppage, whereas the separability index has an obvious decline near the $100^{t h}$ revolution on the curve for the worn tool (Figure $14(\mathrm{~b})$ ). Periodic wear gradually occurs at stages 1,2 and 3 . The longer tooth cuts more material and is more stressed, which causes and aggravates different levels of wear between teeth. From the perspective of the separability index, the correlation continues to decrease and becomes more and more severe. At the start of stage 4, the indicator drops dramatically. It corresponds to the situation where the wear increases exponentially until the tool is completely damaged, which might damage the spindle. After a certain reaction time, the tool was stopped manually (S5), completely withdrew from the material, and reached the idling state $(\mathrm{S} 6)$.

As mentioned in Section 4.3, the separability index $\alpha_{k 1}$ and sensitivity range $\Delta_{W S}$ will fluctuate slightly with different machining conditions. Using Figure 14 (b) as an example, even if the tool continues to wear in the S1, $\mathrm{S} 2$, and S3 stages, the values corresponding to the separability index are all above 0.95 due to its simple rectilinear cutting condition. Therefore, it is difficult to use this value to clearly define the wear status of tools with high precision.

The empirical rule is chosen as the definition of the threshold to solve this problem. The three-sigma interval encompasses $99.7 \%$ of the values under the assumption of a Gaussian distribution [33]. Supposing the operation runs to the $q^{\text {th }}$ revolution, the previous separability indexes from $(q-p)^{t h}$ to $q^{t h}$ revolutions are considered as a cluster to present the current tool state. Their average value $\mu_{q p}$ and standard deviation $\sigma_{q p}$ are calculated as

and

$$
\mu_{q p}=\frac{1}{p+1} \sum_{k=q-p}^{q} \alpha_{k 1}
$$

$$
\sigma_{q p}=\sqrt{\frac{1}{p} \sum_{k=q-p}^{q}\left|\alpha_{k 1}-\mu_{q p}\right|^{2}} .
$$

The value of $p$ can be adjusted to control the response speed of the TCM system. In the studied case, $p=9$, and the thresholds are set at $\mu_{q p} \pm 3 \sigma_{q p}$.

The interval formed by $\mu_{q p} \pm 3 \sigma_{q p}$ for the tool in good condition is very close to the separability index (Figure 14 (a)). The standard deviation $\sigma_{q p}$ emphasizes the variation of the separation index for the worn tool, so regardless of its own value, when $\alpha_{k 1}$ shows a downward trend, $\mu_{q p} \pm 3 \sigma_{q p}$ will form a raised area in both the up and down limit, named the bubble effect.

The vertical diameter of the first bubble in Figure 14 (b) is 0.01196 , and then the diameter gradually increases. At the second and third stages, the values are 0.01898 and 0.03785 , and the fourth stage shows an order of magnitude change at 0.2011 . According to the different accuracy requirements, an alarm threshold can be set corresponding to a suitable bubble 
diameter to monitor the tool state. For the current case, the milling process ideally should be stopped at S2 or S3 to avoid subsequent catastrophic failure. At this time, the tool is no longer in a steady state, which means that at least one of the teeth is entering the rapid wear phase. The result of the verification is in full agreement with the acknowledged tool status.

(ii) TCM based on sensitivity range $\Delta_{W S}$

The assessment based on the sensitivity range has the advantage of skipping the step of milling direction correction (Section 3.2 (iii)). During regular milling conditions, $\Delta_{W S}$ remains stable with a standard deviation of approximately 0.005 . Once tool wear is detected, the sensitivity range varies greatly due to the decline of the correlation between segments. The bubble appears in the same position as with the previous method.

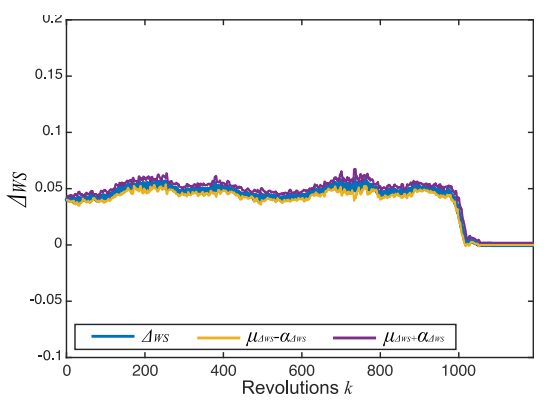

(a)

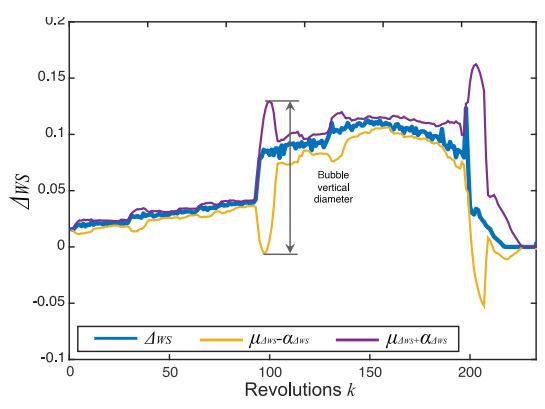

(b)

Fig. 15: Fault detection based on sensitivity range $\Delta_{W S}$ (blue line) with upper (purple line) and lower (yellow line) limit of 3-sigma interval: (a) tool in good condition; (b) worn tool.

The above two judgments are actually two expressions of the same phenomenon. The first one simply relies on the separability index. Only current revolution data need to be included when calculating real-time monitoring, which is fast and efficient. However, the trajectory angle and the spindle angle must be synchronized to achieve higher accuracy. The second method uses the traversal mode, which has the advantage of not requiring a trajectory correction but needs a longer running time to process a larger amount of calculation for the window shift. Depending on the available equipment, it provides different monitoring strategies to suit variable machining conditions.

Note that the signal valley during stoppage will sometimes cross the lower threshold. There is still room for optimization at this stage. The current strategy adopted is to cooperate with the milling program to indicate the finish point of product processing. After the product processing is completed, the monitoring is stopped while the tool is withdrawn (no chips are generated). 
In summary, this method provides effective and stable feedback on the condition of the tool, which is very promising for TCM system construction.

\section{Conclusion}

This article provides a new monitoring solution for monitoring end mill wear in production. The basic principle is to assess the state of the tool by extracting the correlation of the signal corresponding to each tooth. The method relies on the SVD, which benefits from numerically efficient computation. It is shown that each component of the SVD finds a relevant meaning. On this basis, the order separability index is proposed to assess the current operating state of the tool, and two derived strategies are proposed for fault detection.

The method has the advantages of convenience, intuitiveness, and flexibility to adapt to the different milling trajectories (cyclostationary and cyclo-nonstationary conditions). The efficiency and reliability of the method are tested, and the results are promising. In addition, the method may offer potential in the diagnosis of other periodic structures.

\section{Acknowledgement}

This work was performed within the framework of the LABEX CeLyA (ANR10-LABX-0060) of Université de Lyon, within the program Investissements d'Avenir (ANR-11-IDEX-0007) operated by the French National Research Agency (ANR). We would also like to address our special appreciation for the France government fellowship that has provided financial support for this research.

\section{Statements and Declarations}

This study was funded by the France government fellowship. The authors have no relevant financial or non-financial interests to disclose.

We confirm that the manuscript has been read and approved by all named authors and that there are no other persons who satisfied the criteria for authorship but are not listed. We further confirm that the order of authors listed in the manuscript has been approved by all of us.

We confirm that we have given due consideration to the protection of intellectual property associated with this work and that there are no impediments to publication, including the timing of publication, with respect to intellectual property.

\section{References}

[1] A.G. Rehorn, J. Jiang, P.E. Orban, State-of-the-art methods and results in tool condition monitoring: A review. International Journal of Advanced Manufacturing Technology 26, 693-710 (2005) 
[2] M. Lamraoui, M. Thomas, M. El Badaoui, F. Girardin, Indicators for monitoring chatter in milling based on instantaneous angular speeds. Mechanical Systems and Signal Processing 44, 72-85 (2014)

[3] C.H. Lauro, L.C. Brandão, D. Baldo, R.A. Reis, J.P. Davim, Monitoring and processing signal applied in machining processes - A review. Measurement: Journal of the International Measurement Confederation 58, 73-86 (2014). https://doi.org/10.1016/j.measurement.2014.08.035

[4] C. Lauro, L.C. Br, atilde, S.L.M.R. Filho, Monitoring the temperature of the milling process using infrared camera. Scientific Research and Essays 7, 1112-1120 (2013)

[5] S. Dutta, S. Pal, R. Sen, Progressive tool condition monitoring of end milling from machined surface images. Proceedings of the Institution of Mechanical Engineers, Part B: Journal of Engineering Manufacture 232 (2016). https://doi.org/10.1177/0954405416640417

[6] O. Ryabov, K. Mori, N. Kasashima, Laser displacement meter application for milling diagnostics. Optics and Lasers in Engineering 30(3), 251-263 (1998). https://doi.org/https://doi.org/10.1016/ S0143-8166(98)00032-3. URL https://www.sciencedirect.com/science/ article/pii/S0143816698000323

[7] K. Tatar, P. Gren, Measurement of milling tool vibrations during cutting using laser vibrometry. International Journal of Machine Tools and Manufacture 48(3), 380-387 (2008). https://doi.org/https://doi.org/10.1016/ j.ijmachtools.2007.09.009. URL https://www.sciencedirect.com/science/ article/pii/S0890695507001988

[8] Şeref Aykut, E. Bagci, A. Kentli, O. Yazıcıŏ̆lu, Experimental observation of tool wear, cutting forces and chip morphology in face milling of cobalt based super-alloy with physical vapour deposition coated and uncoated tool. Materials \& Design 28(6), 1880-1888 (2007). https: //doi.org/https://doi.org/10.1016/j.matdes.2006.04.014. URL https:// www.sciencedirect.com/science/article/pii/S0261306906001129

[9] M. Shiraishi, Scope of in-process measurement, monitoring and control techniques in machining processes - Part 1: In-process techniques for tools. Precision Engineering 10, 179-189 (1988)

[10] J.D. Kim, D.S. Kim, Development of a combined-type tool dynamometer with a piezo-film accelerometer for an ultra-precision lathe. Journal of Materials Processing Technology 71, 360-366 (1997)

[11] S. Karabay, Design criteria for electro-mechanical transducers and arrangement for measurement of strains due to metal cutting forces acting 
on dynamometers. Materials \& Design 28, 496-506 (2007)

[12] P. Sevilla-Camacho, J. Robles-Ocampo, J. Muñiz, F. Lee-Orantes, Tool failure detection method for high-speed milling using vibration signal and reconfigurable bandpass digital filtering. The International Journal of Advanced Manufacturing Technology 81 (2015). https://doi.org/10.1007/ s00170-015-7302-0

[13] Z. Yuqing, L. Xinfang, L. Fengping, S. Bingtao, X. Wei, An online damage identification approach for numerical control machine tools based on data fusion using vibration signals. Journal of Vibration and Control 21, 2925 -2936 (2015)

[14] P. Sevilla-Camacho, G. Herrera-Ruiz, J. Robles-Ocampo, J. Jauregui, Tool breakage detection in cnc high-speed milling based in feed-motor current signals. The International Journal of Advanced Manufacturing Technology 53, 1141-1148 (2011). https://doi.org/10.1007/s00170-010-2907-9

[15] B. Lee, Application of the discrete wavelet transform to the monitoring of tool failure in end milling using the spindle motor current. The International Journal of Advanced Manufacturing Technology 15, 238-243 (1999)

[16] I. Marinescu, D.A. Axinte, A critical analysis of effectiveness of acoustic emission signals to detect tool and workpiece malfunctions in milling operations. International Journal of Machine Tools and Manufacture 48, $1148-1160$ (2008)

[17] Q. Ren, M. Balazinski, L. Baron, K. Jemielniak, R. Botez, S. Achiche, Type-2 fuzzy tool condition monitoring system based on acoustic emission in micromilling. Information Sciences 255, 121-134 (2014). https://doi. org/10.1016/j.ins.2013.06.010

[18] D. O'Sullivan, M. Cotterell, Temperature measurement in single point turning. Journal of Materials Processing Technology 118, 301 - 308 (2001)

[19] P. Sivasakthivel, R. Sudhakaran, Optimization of machining parameters on temperature rise in end milling of al 6063 using response surface methodology and genetic algorithm. The International Journal of Advanced Manufacturing Technology 67 (2012)

[20] J. Senatore, S. Segonds, W. Rubio, G. Dessein, Correlation between machining direction, cutter geometry and step-over distance in 3-axis milling: Application to milling by zones. Computer-Aided Design 44(12), 1151-1160 (2012). https://doi.org/https://doi.org/10.1016/j.cad. 2012.06.008. URL https://www.sciencedirect.com/science/article/pii/ S0010448512001376 
[21] S. Dutta, A. Kanwat, S. Pal, R. Sen, Correlation study of tool flank wear with machined surface texture in end milling. Measurement 46(10), 4249-4260 (2013). https://doi.org/https://doi.org/10.1016/j. measurement.2013.07.015. URL https://www.sciencedirect.com/science/ article/pii/S026322411300314X

[22] J. Ning, S.Y. Liang, Predictive modeling of machining temperatures with force-temperature correlation using cutting mechanics and constitutive relation. Materials 12(2) (2019). https://doi.org/10.3390/ma12020284. URL https://www.mdpi.com/1996-1944/12/2/284

[23] A.G. Rehorn, E. Sejdić, J. Jiang, Fault diagnosis in machine tools using selective regional correlation. Mechanical Systems and Signal Processing 20(5), 1221-1238 (2006). https://doi.org/https://doi.org/10.1016/j. ymssp.2005.01.010. URL https://www.sciencedirect.com/science/article/ pii/S0888327005000154

[24] E. Sejdic, J. Jiang, Selective regional correlation for pattern recognition. IEEE Transactions on Systems, Man, and Cybernetics - Part A: Systems and Humans 37(1), 82-93 (2007). https://doi.org/10.1109/TSMCA.2006. 886333

[25] P. Niu, Q. Cheng, W. Chang, X. Song, Y. Li, Sensitivity analysis of machining accuracy reliability considering partial correlation of geometric errors for horizontal machining center. Proceedings of the Institution of Mechanical Engineers, Part B: Journal of Engineering Manufacture 235(3), 455-465 (2021). https://doi.org/10.1177/0954405420958843. URL https://doi.org/10.1177/0954405420958843

[26] Anon., Tool life testing in machining — part 2. ISO 8688-2, International Standards Institution (1989)

[27] M. Ritou, S. Garnier, B. Furet, J. Hascoet, Angular approach combined to mechanical model for tool breakage detection by eddy current sensors. Mechanical Systems and Signal Processing 44(1), 211-220 (2014)

[28] W. Kline, R. DeVor, J. Lindberg, The prediction of cutting forces in end milling with application to cornering cuts. International Journal of Machine Tool Design and Research 22(1), 7-22 (1982)

[29] Y. Altintas, Manufacturing Automation, Metal Cutting Mechanics, Machine Tool Vibrations and CNC Design (Cambridge University Press, New York, 2012)

[30] A.G. Asuero, A. Sayago, A.G. González, The correlation coefficient: An overview. Critical Reviews in Analytical Chemistry 36, 41-59 (2006) 
[31] M.E. Wall, A. Rechtsteiner, L.M. Rocha, Singular Value Decomposition and Principal Component Analysis. In: Berrar D.P., Dubitzky W., Granzow M. (eds) A Practical Approach to Microarray Data Analysis (Springer, Boston, MA., 2003), pp. 91-109

[32] F. Girardin, D. Rémond, J.F. Rigal, Tool wear detection in milling - an original approach with a non-dedicated sensor. Mechanical Systems and Signal Processing 24(6), 1907-1920 (2010)

[33] G. Upton, I. Cook, A dictionary of statistics 3e (Oxford university press, 2014) 